\title{
Se former en ligne au Tutorat : un défi pour les assistants-chercheurs
}

\author{
SOPHIE LABRIQUE \\ Conseillère pédagogique au sein du Secteur des Sciences et Technologies, Université \\ catholique de Louvain, Louvain-la-Neuve \\ sophie.labrique@uclouvain.be \\ DELPHINE DUCARME \\ Conseillère pédagogique au sein du Secteur des Sciences et Technologies, Université \\ catholique de Louvain, Louvain-la-Neuve \\ delphine.ducarme@uclouvain.be \\ BENOÎT RAUCENT \\ Professeur à l'École Polytechnique de Louvain et Président du «Louvain Learning Lab », \\ Université catholique de Louvain, Louvain-la-Neuve \\ benoit.raucent@uclouvain
}

\section{Résumé}

L'École Polytechnique de Louvain (EPL) propose depuis de nombreuses années une formation en présentiel pour les étudiants de master. Ils sont appelés les tuteurs et ils encadrent des étudiants de première année dans des enseignements de types : travaux pratiques, apprentissages par problèmes (APP), des projets, apprentissages par exercices (APE), des laboratoires. Une formation en ligne a été créée pour un public d'assistants-chercheurs aux rôles du tuteur. Cette formation hybride intitulée « Tutorer l'Apprentissage en Groupe (TAG) » vise à permettre aux assistants-chercheurs novices et expérimentés de se questionner sur leurs manières d'enseigner pour les améliorer. Nous analysons particulièrement ici les interventions des tuteurs qui encadrent la formation en ligne et leurs impacts pour l'apprentissage. Suite à l'analyse des interventions faites en ligne et en présentiel, et plus précisément en regardant les effets de ces interventions sur le plan métacognitif (réflexivité), nous constatons un besoin patent de la présence du tuteur humain de manière plus régulière au sein de la formation que nous proposons. Ce besoin se manifeste par un sentiment d'isolement de la part des assistants les premières semaines, du besoin de se sentir appartenir à un groupe, de connaître les réflexions des autres pour être motivés à apprendre, et ce, même si tous les assistants évoquent que la 
formation, de par son dispositif, permet très clairement de se questionner sur ses pratiques de tuteur de manière continue pour les améliorer.

\section{Summary}

For many years, Louvain School of Engineering has been offering face-to-face training for master's students. They are called tutors and they supervise first-year students in teaching types: practical work, problem based learning (PBL), projects, learning by exercises, laboratories. An online training has been created for an audience of assistant-researchers in the roles of the tutor. This hybrid course entitled « Tutored learning's group » is designed to allow novice and experienced assistant-researchers to question their ways of teaching to improve them. We analyze here the interventions of the tutors and their impacts for the learning by relying on the notion of tutoring. Following the analysis of the interventions made online and in class, and more specifically by looking at the effects of these interventions on metacognition (reflexivity), we find a crying need for the presence of the human tutor more regularly within the training we offer. This need is manifested by a feeling of isolation on the part of the assistants the first weeks, the need to feel part of a group, to know the reflections of others to be motivated to learn, even if all the assistants evoke that the training, by its device, makes it possible very clearly to wonder about its tutor practices in a continuous way to improve them.

\section{Mots-clés}

Méthodes pédagogiques, accompagnement en ligne, tutorat, réflexivité

\section{Key words}

Teaching methods, online support, tutoring, reflexivity

\section{Introduction et problématique}

De nombreux enseignants du secteur de sciences et technologies de l'Université catholique de Louvain (UCLouvain) ont mis en place de nouvelles approches pédagogiques telles que l'apprentissage par problèmes et/ou par projet, les classes inversées, etc. (Raucent et al., 2014). C'est le cas notamment à l'École Polytechnique de Louvain (EPL) avec l'importante réforme pédagogique introduite en septembre 2000 (Raucent et al., 2004). Le concept central de cette réforme est de placer l'étudiant au centre de sa formation en alternant des activités d'apprentissage autonome et des activités d'apprentissage en groupe en compagnie d'un enseignant (Bouvy et al., 2010). Loin de la simple transmission de savoirs, le rôle de 
l'enseignant devient alors celui de tuteur qui accompagne les étudiants dans leurs cheminements et facilite leurs apprentissages. Ce dernier quitte son rôle de « transmetteur » de savoir pour prendre celui d'accompagnateur des apprentissages des étudiants (Bédard, 2006 ; Bouvy et al., 2010). Ce type de pédagogie nécessite la présence de nombreux encadrants que nous appelons les tuteurs. En effet, l'encadrement d'étudiants en petits groupes d'apprentissage (4 groupes de 6 étudiants dans une salle pour une population de 300 étudiants à l'époque) requiert un nombre élevé d'encadrants. Pour faire face à cette demande, 1'EPL, en plus des enseignants qui encadrent des groupes, propose à des étudiants de master ( $4^{\text {er }}$ et $5^{\text {ème }}$ années d'études) d'encadrer des étudiants de $1^{\text {er }}$ et $2^{\text {ème }}$ années. Ils prennent alors le statut d'étudiantstuteurs. Cette situation connaît deux avantages : elle permet aux étudiants de $1^{\text {er }}$ et $2^{\text {ème }}$ années de disposer d'un encadrement suffisant et de qualité, et aux étudiants-tuteurs d'acquérir des compétences spécifiques en matière de dynamique de groupe. Depuis à peu près quinze ans, les étudiants-tuteurs bénéficient d'une formation pédagogique à la « dynamique de groupe - aux rôles de tuteur » créditée dans leurs programmes de cours.

À l'EPL, les assistants-chercheurs, étudiants en $3^{\text {ème }}$ cycle, encadrent également des étudiants dans des travaux pratiques, des laboratoires, etc. Ils n'avaient jusqu'à présent pas de formation équivalente à celle que l'on propose aux étudiants-tuteurs (de master). Ces deux publics, que nous nommons tous les deux des tuteurs : les étudiants-tuteurs (de master) et les assistantstuteurs (de $3^{\text {ème }}$ cycle) ne sont pas disponibles aux mêmes horaires et notre expérience nous a révélé que les former au sein d'un seul dispositif est peu bénéfique étant donné les expériences et les attentes diverses. Par ailleurs, nous constatons un accroissement du nombre d'assistantstuteurs «novices » qui n'ont pas fait leurs études dans un cadre de pédagogie active et n'ont donc pas pratiqué, ni participé (comme apprenant) à une formation de ce type. Ces nouveaux arrivants ont en général une vision très classique de l'apprentissage. Ils ne savent pas du tout, par exemple, ce qu'est un apprentissage par problèmes (APP). Cette méconnaissance de l'approche pédagogique rend leurs actions difficiles et, en l'absence de référence, ils ont tendance à adopter une attitude décalée par rapport à celle souhaitée, ce qui a un impact assez négatif sur les apprentissages des étudiants. Un fossé de plus en plus grand entre les étudiantstuteurs formés à la pratique du tutorat et les assistants-tuteurs s'est créé. Enfin, les assistantstuteurs doivent répondre à des obligations en termes de recherches à effectuer en vue de réaliser leur doctorat. Ils sont intégrés dans un laboratoire avec des horaires spécifiques, des déplacements fréquents à l'étranger, etc. Il est dès lors difficile de les regrouper selon un agenda 
commun pour assister à des formations en présentiel. Nous sommes ainsi face à un public qui a besoin d'une triple flexibilité : géographique, horaire et pédagogique (Raucent, 2016).

\subsection{Le dispositif}

Depuis 2015, nous avons proposé au public d'assistants-tuteurs une formation en ligne de courte durée basée sur les principes de la formation des étudiants-tuteurs existante, validée sur ces derniers (Sobieski et al., 2016). Cette formation est hybride (Charlier et al., 2006) et en grande partie asynchrone, car les modules peuvent être suivis de manière autonome au rythme de l'apprenant. Elle propose cinq modules d'auto-apprentissage en ligne (Dessus et Marquit, 2009) et deux séances en présentiel (voir schéma 1: organisation de la formation). Les modules en ligne sont organisés selon un même canevas (voir schéma 2 : déroulement de chaque module). À l'issue de la formation, il est attendu de l'assistant-tuteur qu'il soit capable : d'identifier le rôle d'un encadrant en apprentissage actif ; de se questionner et de mettre en cause ses pratiques d'enseignement dans le but de favoriser un apprentissage en profondeur chez les étudiants; de mettre en œuvre des actions pour favoriser l'apprentissage en groupe. Pour participer à cette formation, les assistants-tuteurs doivent encadrer des étudiants dans un enseignement en parallèle de la formation (le même semestre). L'objectif est qu'ils fassent des retours d'expérience de ce qu'ils vivent durant leurs activités de tutorat. Ils effectuent ainsi des allers-retours entre le tutorat et la réflexion sur le tutorat.

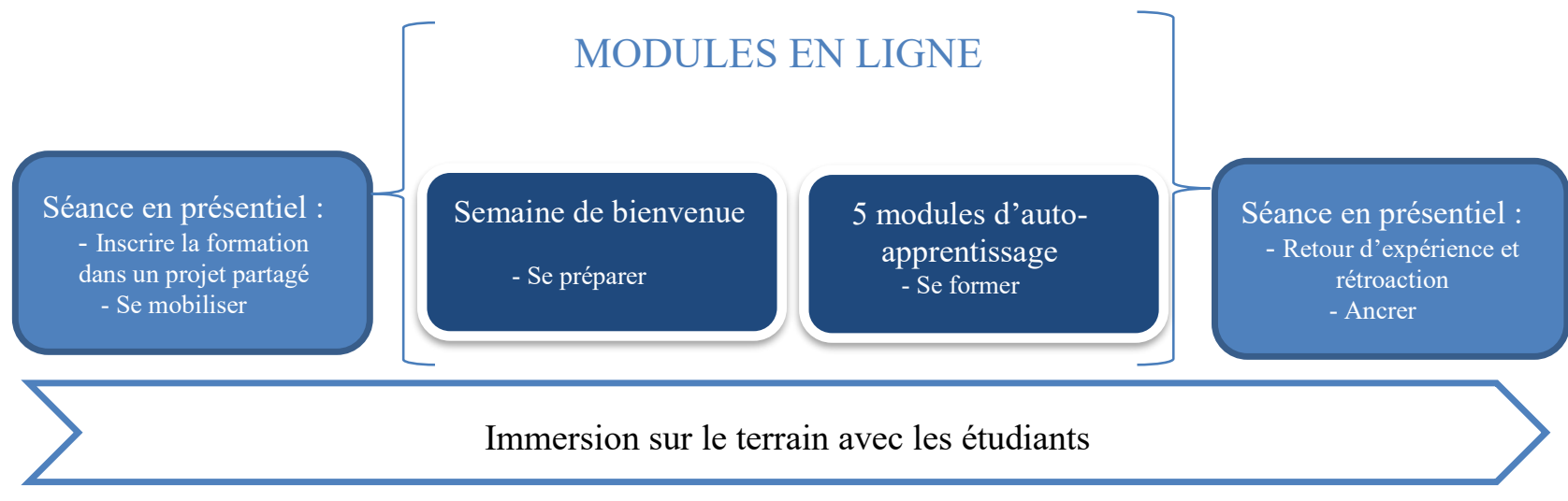

Schéma 1 : organisation de la formation 

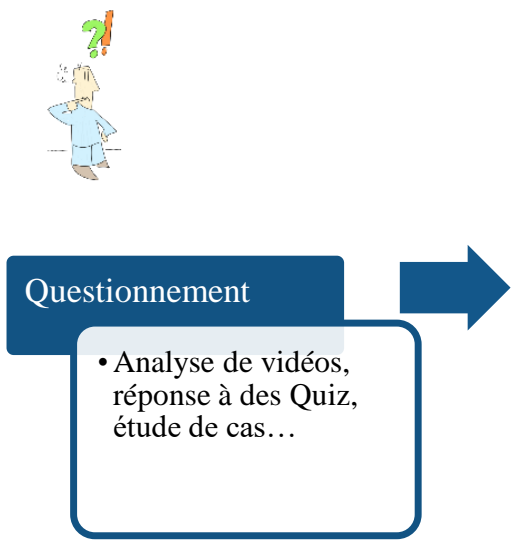
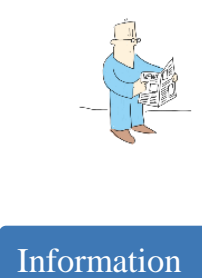

Information

- Textes de la littérature

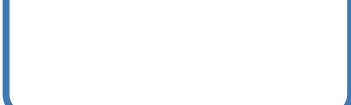

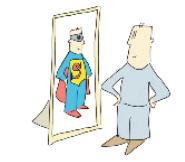

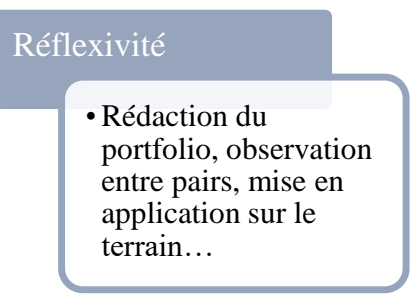

Schéma 2 : déroulement de chaque module

Mais cette formation permet-elle d'atteindre les objectifs visés ? C'est dans cette perspective que nous voulons analyser les effets sur l'apprentissage des interventions des tuteurs que nous sommes et qui ont pour tâche d'encadrer la formation en ligne. Pour nous désigner, nous utiliserons le terme de tuteur-formateur.

Nous voulons analyser d'une part notre dispositif, d'autre part les actions et interventions des tuteurs-formateurs. Dans notre cas, les interventions sont réalisées soit par un robot (un ordinateur dont le dispositif est en ligne sur un site moodle), soit par le tuteur-formateur (en présentiel), soit par les deux. La caractéristique principale des interventions du robot est qu'elle doit être conçue à l'avance par l'humain et programmée pour automatiser l'accompagnement. Il s'agit d'utiliser les critères proposés par Jacques Rodet (2016) pour analyser notre formation pour assistants-tuteurs et offrir aux concepteurs de la formation, des pistes d'amélioration.

Dans un premier temps, nous présentons le cadre de référence. Nous faisons ensuite état des premiers résultats récoltés auprès des assistants-tuteurs de la formation au sujet des actions et interventions des tuteurs-formateurs. Finalement, nous discutons des résultats à la lumière du cadre de référence et nous proposons des pistes d'amélioration du dispositif.

\section{Cadre de référence}

Plusieurs auteurs se sont intéressés à la question de la place de l'humain ou du robot dans l'apprentissage actif. Considérant les recherches menées à ce sujet, nous avons souhaité analyser notre dispositif hybride et les rôles des tuteurs-formateurs. Pour ce faire, nous avons utilisé le cadre de référence de Jacques Rodet (2016). Cet auteur propose une répartition des interventions tutorales entre un robot et des tuteurs humains. Celles-ci sont alors définies en tenant compte des fonctions qui peuvent être effectivement prises par un formateur (robot ou 
humain) : l'accueil, l'organisation, la pédagogie, la motivation, la technique et l'évaluation. Il répartit ensuite les interventions sur les plans de soutien à l'apprentissage : le plan cognitif, le plan socio-affectif, le plan motivationnel et le plan métacognitif. Les fonctions et les plans de soutien à l'apprentissage sont ensuite croisés et mettent en évidence une matrice des interventions des tuteurs-formateurs. Par ce cadre de référence, l'auteur projette que dans certains plans et pour des fonctions particulières, certaines interventions peuvent être assumées par un robot (outil informatique), certaines autres nécessitent des actions coordonnées par un robot et un tuteur humain, et d'autres encore peuvent être confiées uniquement à un intervenant tuteur humain (voir Tableau 1).

Tableau 1 : Répartition des interventions tutorales entre un robot et des tuteurs humains

\begin{tabular}{|c|c|c|c|c|}
\hline Fonctions & Plan cognitif & $\begin{array}{l}\text { Plan } \\
\text { socio-affectif }\end{array}$ & $\begin{array}{l}\text { Plan } \\
\text { motivationnel }\end{array}$ & Plan métacognitif \\
\hline Accueil & $\begin{array}{l}\text { Informer sur le } \\
\text { dispositif }\end{array}$ & $\begin{array}{l}\text { Initier la construction } \\
\frac{\text { d'un sentiment }}{\text { d'appartenance }}\end{array}$ & $\begin{array}{l}\underline{\text { Faire émerger }} \\
\underline{\text { les objectifs }} \\
\text { personnels de } \\
\underline{\text { l'apprenant }}\end{array}$ & $\begin{array}{l}\text { Initier l'apprenant à } \\
\text { faire le point sur ses } \\
\underline{\text { stratégies }} \\
\underline{\text { cognitives }}\end{array}$ \\
\hline Organisation & $\begin{array}{l}\text { Présenter les } \\
\text { méthodologies } \\
\text { appropriées }\end{array}$ & $\frac{\text { Réguler la dynamique de }}{\text { groupe }}$ & $\begin{array}{l}\text { Accompagner } \\
\text { le processus } \\
\text { d'autonomie }\end{array}$ & $\begin{array}{l}\text { Faciliter la } \\
\text { planification } \\
\text { de } \\
\text { l'apprentissage }\end{array}$ \\
\hline Pédagogie & $\begin{array}{l}\text { Apporter des } \\
\text { réponses ou les } \\
\text { susciter. } \\
\text { Remédier }\end{array}$ & $\frac{\underline{\text { Faciliter la }}}{\underline{\text { collaboration des }}}$ & $\begin{array}{l}\underline{\text { Proposer des }} \\
\underline{\text { activités }} \\
\underline{\text { signifiantes }}\end{array}$ & $\begin{array}{l}\frac{\text { Susciter l'expression }}{\text { critique sur le }} \\
\underline{\text { dispositif }}\end{array}$ \\
\hline $\begin{array}{l}\text { Socio- } \\
\text { affectif }\end{array}$ & $\begin{array}{l}\text { Personnaliser le } \\
\text { soutien à } \\
\text { l'apprentissage }\end{array}$ & $\begin{array}{l}\text { Rompre l'isolement de } \\
\text { l'apprenant }\end{array}$ & $\begin{array}{l}\text { Lutter contre } \\
\text { l'abandon }\end{array}$ & $\begin{array}{l}\text { Faire prendre } \\
\text { conscience de ses } \\
\text { habiletés à } \\
\text { collaborer }\end{array}$ \\
\hline Technique & $\begin{array}{l}\text { Aider à } \\
\text { maîtriser } \\
\text { l'environnement } \\
\text { d'apprentissage }\end{array}$ & $\begin{array}{l}\text { Susciter l'entraide } \\
\underline{\text { technique entre }} \\
\underline{\text { apprenants }}\end{array}$ & $\begin{array}{l}\text { Encourager } \\
\text { l'utilisation des } \\
\text { outils }\end{array}$ & $\begin{array}{l}\frac{\text { Susciter la prise de }}{\text { distance réflexive }} \\
\underline{\text { sur les usages des }} \\
\underline{\text { outils }}\end{array}$ \\
\hline Evaluation & $\begin{array}{l}\text { Annoncer } \\
\text { clairement les } \\
\text { critères } \\
\text { d'évaluation }\end{array}$ & $\begin{array}{l}\text { Produire des } \\
\text { rétroactions à portée } \\
\text { formative }\end{array}$ & $\begin{array}{l}\text { Encourager et } \\
\text { féliciter }\end{array}$ & Aider à s'auto-évaluer \\
\hline
\end{tabular}

(Rodet, 2016)

Légende : En gras, les interventions pouvant être confiées à un robot ayant été préalablement formé ; en italique, les interventions partagées par un robot et les tuteurs humains; en souligné, les interventions ne pouvant être confiées qu'à des tuteurs humains. 
Nous nous intéresserons particulièrement aux actions et interventions sur le plan métacognitif, ce qui nous permettra de poser un regard sur nos objectifs de formation, à savoir, provoquer de la réflexivité chez les formés (assistants-tuteurs).

\section{Méthodologie}

Considérant que nous nous intéressons à la question des interventions dans le dispositif de formation destiné aux assistants-tuteurs, nous décrivons très brièvement dans un premier temps notre dispositif au regard du tableau de Rodet (2016). Par la suite, nous présentons une analyse critique du dispositif du point de vue des formés (assistants-tuteurs), toujours en regard du cadre théorique.

\subsection{Description du dispositif au regard du cadre théorique}

Les participants sont informés de l'organisation de la formation, des méthodologies utilisées, des techniques, des objectifs et de l'évaluation grâce à l'action du robot. Dans notre cas, il s'agit d'une courte vidéo et de quelques textes assez brefs.

Sur le plan socio-affectif et motivationnel, notre dispositif prévoit l'intervention humaine (des tuteurs-formateurs) (souligné dans le Tableau 1). En effet, nous avons fait le choix de prévoir deux séances en présentiel dans cette formation qui encadrent les modules en ligne. La première (en début de dispositif) vise la création d'une communauté d'apprentissage et d'un sentiment d'appartenance. Elle a également pour but de faire émerger les attentes et motivations des participants à suivre cette formation. La seconde séance en présentiel (en fin de dispositif) vise à enrichir davantage le plan métacognitif. Elle est en quelque sorte l'aboutissement des réflexions, questionnements de chaque individu. Le formateur invite le participant à faire le point sur ses réflexions en confrontant ses idées à celles des autres participants, ce qui, nous pensons, peut avoir un effet sur la motivation et sur le plan métacognitif. Pour ce qui est de faire le point sur ses stratégies cognitives (voir Tableau 1 : fonction d'accueil sur le plan métacognitif) énoncé par Rodet (2016) comme étant une intervention unique du tuteur humain, nous pensons que l'intervention du robot peut aussi aider les participants dans leurs apprentissages. Dans notre dispositif, pour chaque thème, le participant est d'abord invité à se questionner et à faire émerger ce qu'il sait sur celui-ci à l'aide de questionnaire, vidéos, quiz... (voir Schéma 2). Lors de la seconde séance en présentiel, cet objectif est aussi atteint et est 
amené par l'assistant-tuteur et par les pairs. Dans notre dispositif, cette intervention est donc menée conjointement par le robot et l'humain.

Concernant les interventions partagées par un robot et les tuteurs humains (en italique dans le Tableau 1), notre dispositif prévoit que le binôme robot-humain intervienne à plusieurs reprises sur différents plans. Les interventions les plus significatives étant pour nous la personnalisation de l'apprentissage et la rétroaction individuelle donnée aux assistants-tuteurs qui leur permettent de s'auto-évaluer. Sur le plan motivationnel, le robot et les tuteurs humains devraient accompagner à l'autonomie.

\subsection{Démarche d'évaluation du dispositif}

Après avoir assuré la concordance de notre dispositif avec le cadre théorique, nous souhaitions que les assistants-tuteurs en évaluent la valeur à l'aide d'un questionnaire de satisfaction qui demande aux huit participants de se positionner sur une échelle d'accord à quatre niveaux ( 0 à 4: de totalement en désaccord à totalement d'accord). Ce questionnaire comportait sept questions portant sur l'appréciation générale de la formation; la satisfaction de l'encadrement lors de la formation; la satisfaction du moment de la formation dans le semestre ; la satisfaction du caractère «en ligne » de la formation ainsi que des deux modules en présentiel; la satisfaction concernant les travaux à remettre. Nous leur avons également demandé, via le questionnaire, leur sentiment sur leur évolution en termes d'interventions tutorales : rendre ses étudiants actifs, réagir de manière pertinente, intervenir pour favoriser un apprentissage en profondeur chez les étudiants. Des questions portaient également sur le ressenti de leurs rôles de tuteur : ont-ils pu questionner leurs propres pratiques ? Ont-ils utilisé les rôles du tuteur dans les groupes d'apprentissage?

En plus de ce questionnaire, nous avons eu une discussion (entretien semi-directif filmé). Les questions portaient sur les points forts et les points à améliorer du dispositif en mettant en évidence l'impact de la formation en ligne asynchrone et des interventions des tuteursformateurs lors des séances en présentiel. Sur les 8 participants sollicités, 5 ont répondu au questionnaire en ligne et 8 étaient présents à la séance de discussion. Le questionnaire et les questions posées lors de la discussion sont disponibles en annexe.

La section ci-dessous présente quelques éléments de synthèse sur base des résultats recueillis. Nous les classons selon le cadre théorique : ce qui concerne les interventions du robot (moodle), les interventions qui concernent les tuteurs-formateurs, et celles qui concernent les deux. 


\section{Résultats}

Pour répondre aux questions de recherche, nous présenterons d'abord les résultats portant sur l'objectif de favoriser la réflexivité chez les assistants-tuteurs au sein de la formation. Ensuite, nous nous intéressons aux compétences dans les rôles du tuteur et ce qui les renforcent. Finalement, nous présenterons les résultats portant sur les effets des rétroactions en ligne de notre part, tuteurs-formateurs.

Si notre objectif est d'amener les assistants-tuteurs à se poser des questions sur leurs pratiques pour les améliorer, alors que la plupart des modules se déroulent en ligne, ainsi qu'à réaliser des interventions qui provoquent la réflexion malgré qu'elles soient données à distance, nous pouvons observer différents résultats. Les assistants-tuteurs ont évoqué l'importance pour leurs apprentissages, de donner dans le même temps que la formation, les séances avec les étudiants. «Faire des allers-retours entre les modules et la pratique de terrain nous permet de renforcer nos démarches réflexives » (propos des assistants-tuteurs). Ils mentionnent que c'est aidant de devoir se questionner sur une thématique pédagogique par l'expérience qu'ils possèdent, de bénéficier ensuite d'une restructuration théorique à l'aide des vidéos en ligne ou des textes de la littérature. Ils citent que suite à cela, ils se questionnent à nouveau sur ce qu'ils pourraient améliorer dans leurs pratiques en les formalisant sur le moodle (dans le Portfolio) et que suite aux rétroactions fournies par les tuteurs-formateurs, leurs questionnements continuent. «Tester sur le terrain et faire le point ensuite sur ce qui a marché ou non et pourquoi, pour se projeter pour une nouvelle amélioration est intéressant, c'est un processus continu de questionnement » (propos d'un assistant-tuteur). Nous analysons au regard de leurs propos, que leurs interventions en tant que tuteur humain, en présentiel avec leurs propres étudiants, renforcent la prise de distance réflexive sur leurs propres actions de tuteurs et l'auto-évaluation de leurs pratiques pour l'apprentissage. Ces allers-retours entre la formation et la pratique de terrain, ils les considèrent comme essentiels dans le dispositif.

En outre, un assistant-tuteur nous révèle qu'il ne s'est pas senti armé pour agir et réagir de manière pertinente face à son groupe d'apprenants. Il mentionne les difficultés à utiliser les différents rôles de tuteur en séance. Les quatre autres assistants-tuteurs quant à eux disent que la formation leur a permis de rendre leurs étudiants actifs, d'agir de manière pertinente face à leurs groupes d'apprenants, et de mettre en place des interventions qui stimulent les apprentissages en profondeur chez les étudiants. Nous l'anticipions, le renforcement de la dimension humaine du dispositif par les séances avec les étudiants en présentiel a un impact 
certain sur les apprentissages des assistants-tuteurs à leurs rôles. Parallèlement à cela, pour soutenir leurs propres apprentissages dans les rôles de tuteur et pour faire davantage de lien entre leurs pratiques et les concepts vus dans la formation «TAG», certains assistants-tuteurs souhaitent également plus d'encadrement et d'accompagnement en présentiel de notre part (tuteurs-formateurs). "Une séance en présentiel supplémentaire en début de formation pour faire le point et discuter de nos interventions avec les autres assistants-tuteurs et avec les tuteurs-formateurs nous permettrait de devenir plus performants dans nos attitudes et actions de tuteur avec nos étudiants » (propos d'un assistant-tuteur).

Une de nos missions de tuteur-formateur fut de donner une rétroaction de qualité, en ligne, sur les réflexions et actions des assistants-tuteurs. Celle-ci a pour but de favoriser les interactions et les échanges, sans que ce soit un retour transmissif. Suite à l'analyse des résultats, il importe de mettre en évidence une demande de certains assistants-tuteurs (quatre formés), d'ajout, en plus de la rétroaction individuelle personnalisée, d'une rétroaction collective en ligne qui fait état en quelques phrases des éléments de réflexivité, des questionnements communs à tous les participants. «On reçoit une rétroaction mais on ne sait pas du tout où en sont les autres participants dans leurs réflexions, on ne sait pas si on est tout à fait décalé ou si on se pose tous le même genre de questions » (propos d'un assistant-tuteur). «Les premières semaines, on se sentait isolé » (propos d'un assistant-tuteur). Ils évoquent ici le besoin d'appartenir à un groupe d'apprentissage, de collaborer pour leurs propres apprentissages et pour être motivés à apprendre. Ils expriment : «nous avons besoin de savoir si nous nous posons les mêmes questions ou si nous sommes tout à fait décalés par rapport au groupe ». «C'est plus motivant de savoir que nous ne sommes pas seuls dans nos apprentissages et dans nos réflexions » (propos d'un assistant-tuteur). Ces ressentis, les assistants-tuteurs l'expliquent par le fait que la formation est en ligne, qu'ils se retrouvent seuls devant leurs écrans d'ordinateur. Ils évoquent l'importance pour la motivation à apprendre, de savoir où en sont les autres participants dans leurs réflexions, leurs apprentissages, leurs questionnements. Notre dispositif prévoit que l'action du robot (moodle) et du tuteur humain permet aux participants de recevoir une rétroaction personnalisée en ligne, ce qui permet des interactions entre l'assistant-tuteur et le tuteur humain, mais cela semble ne pas suffire. Donner une rétroaction individuelle ne semble pas initier la construction d'un sentiment d'appartenance, réguler la dynamique de groupe et faciliter la collaboration. Ce sentiment éprouvé par certains, nous aurions pu l'anticiper, considérant que ces fonctions devraient principalement et uniquement être dévolues au tuteur humain, le tuteur-formateur, et non le robot. De cela, il en découle une demande de la part de 
certains participants ( 2 sur les 8 ) d'une séance supplémentaire en présentiel après 3 ou 4 semaines de travail en ligne.

\section{Bilan et perspectives}

Dans une optique d'étude des interventions des tuteurs-formateurs et des effets de la formation hybride «tutorer l'apprentissage en groupe (TAG)» sur la réflexivité et le plan métacognitif des assistants-tuteurs de l'EPL, notre étude a permis de cerner les points positifs et les éléments à améliorer identifiés par les assistants-tuteurs, participant au dispositif de formation.

Les résultats de l'analyse présentés dans cet article sont l'aboutissement d'une première expérimentation et concernent un petit nombre d'assistants-tuteurs. Ils sont encourageants car ils montrent que le dispositif a contribué effectivement à les former à leurs rôles de tuteur. Les participants apprécient l'aspect en ligne et asynchrone des cinq modules mais regrettent le manque de collaboration entre eux et avec les formateurs. Un assistant mentionne également que la formation ne l'a que partiellement aidé à se sentir compétent pour agir de manière pertinente dans les différentes situations d'apprentissage avec ses étudiants. Les démarches d'interventions des tuteurs humains et/ou robot ont leurs objectifs spécifiques et contribuent à l'apprentissage sur différents plans et pour diverses fonctions (Rodet, 2016). Dans notre dispositif, le robot intervient en complément du tuteur humain et les résultats montrent que ce dernier conserve une vraie plus-value. En effet, les interventions qui ont un impact sur la motivation, le socio-affectif et le métacognitif, restent du domaine exclusif des tuteurs humains dans la mesure où elles demandent une compréhension affective, émotionnelle du besoin d'accompagnement du participant formé (Rodet, 2016). Les résultats ont amené de nombreux questionnements sur les rôles des intervenants. La rétroaction donnée par le tuteur-formateur dans le moodle n'est-elle pas trop transmissive puisqu'elle est en ligne ? Permet-elle la réflexivité de nos assistants-tuteurs ? Si notre dispositif avait été totalement dispensé en présentiel, aurions-nous de meilleurs assistants-tuteurs?

Pour favoriser la réflexivité de nos assistants-tuteurs, dans un premier temps, nous allons faire en sorte que les rétroactions personnalisées puissent engendrer davantage d'interactions entre eux. Nous pourrions par exemple leur proposer de se donner eux-mêmes des rétroactions en plus des nôtres (peer review). Ceci favoriserait les échanges, les réflexions et la motivation (Daele et Lambert, 2013). Ensuite, nous ambitionnons de rédiger une rétroaction commune après chaque module de formation, et de l'envoyer à tous, ce qui permettrait de mettre en 
évidence les points de réflexion communs. Cette rétroaction collective pourrait favoriser le sentiment d'appartenance, la communauté d'apprentissage et les encouragerait, les motiverait dans leurs apprentissages.

Nous nous questionnons également sur l'idée d'ajouter une séance en présentiel au milieu de la formation. Nous pensons qu'elle permettrait la création d'un vrai groupe d'apprentissage, et audelà, de lancer les assistants-tuteurs dans le contenu de la formation. Nous pensons que partager ses questionnements en confrontant ses idées et en les exposant favoriseraient la réflexivité de chacun.

Des analyses complémentaires vont prendre cours prochainement et pourront bonifier nos premiers résultats et nous permettre de répondre à certaines de nos interrogations. Notre questionnement principal porte sur le sentiment «d'isolement » (manque de coopération entre les formés et manque de rétroaction relative pour se situer par rapport aux autres). Dans cet esprit, nous avons récemment mis en place un mécanisme d'apprentissage et d'évaluation par les pairs en ligne et en présentiel par le biais d'une observation en séance avec les étudiants. En plus, nous avons organisé un nouveau module qui aborde la question de comment fournir une rétroaction constructive aux étudiants. Ces nouveaux dispositifs feront l'objet d'une publication future.

Nous ambitionnons que cette formation réduise l'écart entre des encadrements donnés par les tuteurs-étudiants (de master) et les assistants-tuteurs ou, à tout le moins, d'amenuiser les différences entre tuteurs et de donner l'impression aux étudiants d'une équité pédagogique. Qu'ils soient encadrés avec le même niveau de qualité, sans craindre d'avoir reçu une formation plus ou moins bonne à cause de l'intervenant.

\section{Références bibliographiques}

Bédard, D. (2006). Comment former les étudiants et les enseignants à leurs nouveaux métiers ? Dans B. Raucent et C. Vander Borght, Être enseignant, Magister? Metteur en scène? (p. 301360). Bruxelles : De Boeck.

Bouvy, T., De Theux, M.-N., Raucent, B., Smidts D., Sobieski, P. et Wouters, P. (2010). Compétences et rôles du tuteur en pédagogies actives. Dans B. Raucent, C. Verzat et L. Villeneuve, Accompagner les étudiants : Quels rôles pour l'enseignant ? Quels dispositifs ? Quelles mises en æuvre? (p. 371-396). Bruxelles : De Boeck.

Charlier, B., Deschryver, N. et Peraya, D. (2006). Apprendre en présence et à distance, une définition des dispositifs hybrides. Distances et Savoirs, 4(4), 469-496. 
Daele, A. et Lambert, M. (2013). Comment fournir un feed-back constructif aux étudiants ? Dans D. Berthiaume, et $\mathrm{N}$. Rege Colet, La pédagogie de l'enseignement supérieur : repères théoriques et applications pratiques (p. 299-312). Berne : Peter Lang.

Dessus, P. et Marquet, P. (2009). Les effets des dispositifs d'enseignement à distance. Distances et Savoirs, 7(1), 1-7.

Le Boterf, G. (2010). Le principe de réflexivité. Dans B. Raucent, C. Verzat et L. Villeneuve, Accompagner les étudiants : Quels rôles pour l'enseignant? Quels dispositifs ? Quelles mises en æuvre? (p. 155-185). Bruxelles : De Boeck.

Paquay, L., Van Nieuwenhoven, C. et Wouters, P. (2010). L'évaluation, levier de développement professionnel ? Bruxelles : De Boeck.

Raucent, B. (dir.). (2016). Les cahiers du Louvain learning Lab : Carnet de l'enseignant. Louvain-la-Neuve : Université catholique de Louvain.

Raucent, B., Braibant, J.-M., de Theux, M.-N., Jacqmot, C., Milgrom E., Vander Borght, C. et Wouters, P. (2004). Devenir ingénieur par apprentissage actif : compte rendu d'innovation. Didaskalia, 24, 81-101.

Raucent, B., Milgrom, E. et Romano, C. (2014). Guide pratique pour une pédagogie active : les APP..., Apprentissages par Problèmes et par Projet. Toulouse et Louvain : INSA Toulouse et École Polytechnique de Louvain.

Raucent, B. et Vander Borght, C. (2006). Être enseignant, Magister ? Metteur en scène? Bruxelles : De Boeck.

Rodet, J. (2016). L'ingénierie tutorale : Définir, concevoir, diffuser et évaluer des services d'accompagnement des apprenants d'un digital learning. Paris : Jip.

Sobieski, P., Ducarme, D., Wertz, V. et Raucent, B. (2016). FSA2351 : notes du cours [présentations PowerPoint]. Repéré dans dynamique de groupe, rôles du tuteur : https://moodleucl.uclouvain.be/ 
Annexe

Les questions que nous avons posées aux participants en entrevue sont :

a) Si vous deviez énoncer une chose à garder dans la formation, quelle serait-elle ?

b) Donnez un élément qui vous a manqué durant la formation

c) Si vous deviez décrire en trois mots la formation, quels seraient-ils ?

Les résultats au questionnaire :

\section{ÉVALUATION DU DISPOSITIF HYBRIDE «TUTORER L'APPRENTISSAGE EN GROUPE»}

\begin{tabular}{llllll}
0 & 0 & 1 & 2 & 3 & 4 \\
\hline
\end{tabular}

J'estime que la formation m'a aidé à :

[questionner mes pratiques d'encadrant]

[utiliser les différents rôles d'un encadrant (CQFD) dans..

[rendre mes étudiants actifs]

[agir et réagir de manière pertinente face à un groupe...

[mettre en place des interventions qui stimulent...

Je suis satisfait de l'encadrement lors de cette formation.

Je trouve utile que la formation se déroule au même...

Je suis satisfait que la formation se soit déroulée.

Je suis satisfait des deux modules en présentiel.

Les travaux/quiz à rendre et compléter en fin de module... 